[0212-7199(2001) 18: 1; pp 38-44 ANALES DE MEDICINA INTERNA Copyright $\odot 2001$ ARAN EDICIONES, S.A.

AN. MED INTERNA (Madrid) Vol. 18, N. $^{\circ} 1$, pp. $38-44,2001$

\title{
Distrofia simpático refleja: aspectos psicológicos y psicopatológicos
}

\author{
C. BRUSCAS IZU, M.J. PÉREZ-ECHEVERRÍA*, M. MEDRANO SAN ILDEFONSO, \\ S. HIJOS*, L. SIMON
}

Servicio de Reumatología. Hospital Lapeyronie. Montpellier. Francia. *Servicio de Psiquiatría. Hospital Miguel Servet. Zaragoza

REFLEX SYMPATHETIC DYSTROPHY: PSYCHOLOGICAL AND PSY CHOPATHOLOGICAL ASPECTS

\begin{abstract}
RESUMEN
Clásicamente se ha admitido un terreno psicológico predisponente en la aparición de la distrofia simpático refleja. No obstante, no hay unanimidad en esta apreciación ya que existen estudios que abogan por el carácter reactivo de la afectación psicológica en la distrofia simpático refleja. En el presente trabajo revisamos la literatura publicada al respecto, discutimos sobre los test de evaluación psicológica utilizados hasta el momento, y planteamos la posibilidad de utilizar otros de más fácil manejo e interpretación.
\end{abstract}

PALABRAS CLAVE: Distrofia simpático refleja. Algodistrofia. Dolor. Psicología. Psiquiatría.

\begin{abstract}
It has traditionally been accepted that a predisposing psychological field exists with the appearance of the reflex sympathetic dystrophy. Nevertheless, there is no unaminous agreement in this interpretation, since surveys exist wich are in favour the reactive character of the psychological effect on reflex sympathetic dystrophy. In this study, we revise literature already published on the subject, present arguments on the psychological evaluation tests carried out to date and propose the possibility of using other test wich are easier to manage and to interpret.
\end{abstract}

KEY WORDS: Reflex sympathetic dystrophy. Algodystrophy. Pain. Psy chology. Psychiatry.

Bruscas Izu C, Pérez-Echeverría MJ, Medrano San Ildefonso M, Hijos S, Simon L. Distrofia simpático refleja: aspectos psicológicos y psicopatológicos. An Med Interna (Madrid) 2001; 18: 38-44.

\section{INTRODUCCIÓN}

La distrofia simpático refleja (DSR) es una patología reumatológica difícil de definir, puesto que no hay una sino varias formas clínicas de presentación. En general, agrupa un grupo de entidades que suelen tener una serie de características etiológicas, clínicas, biológicas, radiológicas y gammagráficas comunes. Clínicamente consta de una fase caliente con dolor y signos clínicos pseudoinflamatorios que puede sucederse de una fase fría con disminución del dolor, de los signos clínicos pseudoinflamatorios y con aumento progresivo de la impotencia funcional. En ocasiones, puede aparecer una tercera fase correspondiente a las secuelas. Esta descripción clínica es más propia de las localizaciones distales. Radiológicamente suele haber una desmineralización ósea tardía y en la gammagrafía ósea podemos encontrar una hiperfijación precoz, aunque se admiten las formas hipofijantes. En la analítica no se aprecian parámetros inflamatorios, destacando una VSG normal. Su diagnóstico se realiza según los crite- rios descritos por Doury (Tabla I); si bien, otros autores asumen los de Kozin. La evolución es generalmente favorable, salvo algunos casos que pueden dejar secuelas (1-6).

La patogenia de la DSR se ha basado, tradicionalmente, en la teoría de Leriche (7), según la cual habría una agresión nociceptiva, anormalmente persistente, que desencadenaría una perturbación del sistema simpático que condicionaría, a su vez, una alteración microcirculatoria periférica. No obstante, esta teoría no llega a explicar todas las formas de presentación clínica de la DSR. Recientemente, se ha cuestionado dicha teoría y se ha involucrado en la patogenia a ciertos neuromediadores, liberados por fibras aferentes polimodales $\mathrm{C}$, que serían responsables de la alteración microvascular y nociceptiva (8-11).

En ocasiones, la DSR se presenta sin una causa aparente, catalogándose como idiopática pero, generalmente, la DSR es secundaria a alguna circunstancia desencadenante o factor predisponente, habiendo incluso cierta relación loco-regional. En este sentido, se observa que muchas DSR, además de una

Trabajo aceptado: 20 de Diciembre de 1999

Correspondencia: C. Bruscas Izu. Avda. Goya, 5. ${ }^{\circ}$ D - 50005 Zaragoza 


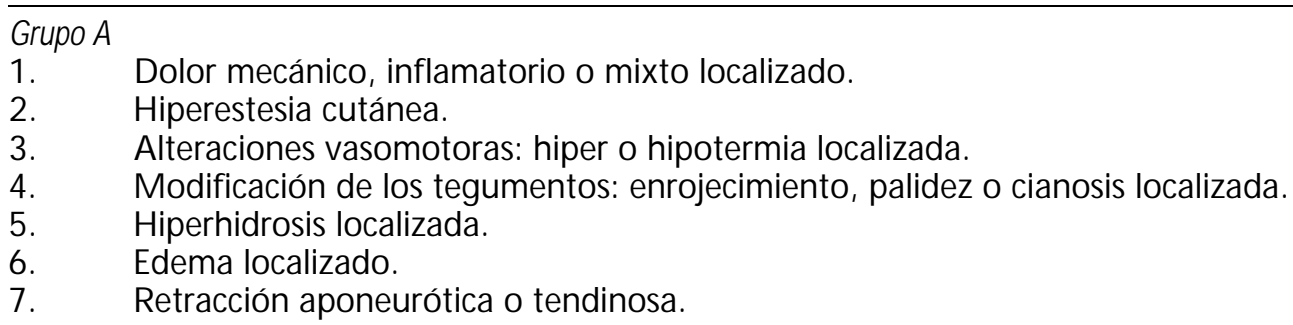

Grupo B

1. Desmineralización ósea homo o heterogénea localizada sin osteocondensación ni alteración de la interlínea.

2. Hiper o hipocaptación ósea localizada en la gammagrafía ósea.

Grupo C

1. Ausencia de signos biológicos de inflamación (VSG normal).

Grupo D

1. Líquido articular no inflamatorio.

2. Histología sinovial con signos de congestión vascular y sin infiltrados inflamatorios.

3. Histología ósea normal o con rarefacción de las trabéculas o con hiperactividad osteoclástica o hiperactividad osteoblástica.

Grupo E

1. Eficacia espectacular de la calcitonina, beta bloqueantes o bloqueos simpáticos.

Diagnóstico cierto: a) al menos 1 criterio de cada grupo $A, B$ y $C$; b) al menos 1 criterio de cada grupo $A, C$ y $E$.

Diagnóstico probable: a) 1 criterio de cada grupo B, C y D; b) 2 criterios del grupo A y al menos 1 criterio de los grupos $C$ y $D$; c) 2 criterios del grupo A y el criterio del grupo $\mathrm{E}$.

Diagnóstico posible si 1 criterio de cada uno de los grupos A y B o A y C o A y E o B y C.

causa desencadenante o sin ella, presentan un terreno psicológico peculiar que habitualmente se ha considerado como predisponente (12-15).

En el presente trabajo discutimos sobre el terreno psicológico que acompaña a la DSR y revisamos la literatura publicada al respecto. En concreto, comentamos los instrumentos de evaluación más utilizados hasta el momento en la patología que nos ocupa, haciendo hincapié en los trabajos más reseñables, y planteamos la posibilidad de utilizar otros test y/o cuestionarios de valoración psicoterápica que pudieran utilizarse en estos pacientes por su fácil manejo y sencilla interpretación.

\section{DISCUSIÓN}

En general, podemos encontrar diferentes conexiones entre las enfermedades reumatológicas y el terreno psicológico. En primer lugar, se pueden observar pacientes en los que el trastorno fundamental es la presencia de un problema psíquico y como consecuencia del cual el paciente somatiza en forma de proceso reumatológico, caracterizado fundamentalmente por dolor, generalizado o localizado, en estructuras predominantemente musculares y que se conoce como reumatismo psicógeno. Otro grupo de pacientes presenta trastornos músculo-esqueléticos de partes blandas para los que tienen una predisposición física o genética, acompañándose, como factor desencadenante inicial o concomitante, de un trastorno psíquico. Finalmente, existe un último grupo de padecimientos reumatológicos en los que la presencia de un trastorno psíquico es reactivo y secundario a la enfermedad orgánica, debido a la cronicidad de la misma y al dolor, incapacidad y deformidades que ésta ocasiona (16).

En lo que respecta a la DSR, se ha admitido que las personas afectadas de una DSR son "psíquicamente peculiares", apreciándose en su interrogatorio como emotivas, nerviosas, irritables, ansiosas, etc (1). Esta circunstancia no se asocia solamente al adulto sino que también se ha relacionado con la DSR del niño y del adolescente (17-24). Dicho psiquismo peculiar se ha considerado, clásicamente, un terreno predisponente de la DSR como queda reflejado en diferentes trabajos (25-31). No obstante, existen estudios más actuales que cuestionan la metodología de los trabajos anteriores y, por tanto, dicha predisposición. Estos estudios abogan por el carácter reactivo de la afectación psicológica a la DSR (15).

No existen muchos trabajos que hagan referencia al psiquismo y la DSR y en ellos la cifra de prevalencia psicoterápica documentada es muy variable, oscilando entre un $11 \%$ y un $80 \%$ (15,25-39). Esta variabililidad está probablemente en función de las diferentes poblaciones y metodologías empleadas en los diversos estudios. De todos los trabajos, destaca el de Acquaviva (12) por su amplia casuística (765 casos); encontrando en un $26 \%$ de las DSR un terreno piscológico alterado. 


\section{TEST UTILIZADOS CLÁSICAMENTE}

El primer trabajo que aborda la posible relación entre las alteraciones psicológicas y la DSR, aplicando el Minnesota Multiphasic Personality Inventory o MMPI, fue realizado por la escuela de Montpellier en 1981 (32). Este test, introducido por Hathaway (40) en 1943, es probablemente el cuestionario autoadministrado para valoración psicométrica de la personalidad más ampliamente difundido que existe.

Para realizarlo simplemente se requiere saber leer y tener más de 16 años. Consiste en un cuestionario de más de 500 preguntas sobre la presencia o ausencia de sentimientos, pensamientos y experiencias. A estas preguntas hay que responder con "verdadero", "falso" o "no sé".

El MMPI explora los siguientes componentes de la personalidad: hipocondría (Hs), depresión (D), histeria (Hy), desviación psicótica (Pd), masculinidad-feminidad (Mf), paranoia $(\mathrm{Pa})$, psicastenia $(\mathrm{Pt})$, esquizofrenia $(\mathrm{Sc})$, manía $(\mathrm{Ma})$ e introversión social ( $\mathrm{Si}$ ). Presenta también 4 escalas de corrección que analizan la fiabilidad y sinceridad y que ofrecen información sobre el estilo de respuesta del sujeto evaluado. Además, la versión española incluye otras escalas adicionales como fuerza del yo, dependencia, dominancia, responsabilidad y control.

El cuestionario puede arrojar los siguientes perfiles básicos: psicótico, neurótico y psicopático.

El MMPI tiene la ventaja de su sencillez, hasta tal punto que cualquier persona podría aplicarlo sin necesidad de ser psiquiatra o psicólogo. No obstante, su corrección es compleja ya que los resultados de las escalas deben interpretarse en relación al perfil global y, por otra parte, puede llegar a cansar al paciente por la cantidad de preguntas.

En la práctica, ha demostrado su utilidad clínica en la psiquiatría, en la medicina forense (campo de peritación psiquiátrica), en la valoración del dolor crónico y en la orientación y selección profesional $(41,42)$.

Este cuestionario se ha aplicado en muchos de los trabajos realizados para valorar la posible alteración psicológica en el curso de la DSR. Así, Pelissier (35) aplica el MMPI en 31 pacientes afectos de una DSR, encontrando un perfil psicológico alterado en un $61,2 \%$ de ellos; siendo, por orden de frecuencia, la depresión, la histeria y la psicastenia las alteraciones más comúnmente encontradas. De este modo, para Pelissier la personalidad del paciente con DSR tendría elementos obsesivos (duda, ambivalencia, perfeccionismo) que se mezclarían con elementos histéricos (tendencia a somatizar un conflicto interior), mientras que el componente depresivo sería probablemente reaccional a la DSR. Para el autor las alteraciones encontradas no son patognomónicas de la DSR puesto que similares anomalías pueden encontrarse en el imsomnio. Por contra, estas alteraciones diferirían de las observadas en estudios realizados en población afectada de lumbalgia crónica en la que domina la hipocondría, la depresión y la histeria. Finalmente, Pelissier sugiere el tratamiento de estas alteraciones con psicoterapia de relajación como complemento al tratamiento habitual de la DSR. En este sentido, en un trabajo realizado en nuestro Servicio de Reumatología (2), sobre una población de 44 pacientes diagnosticados de una DSR y aplicando el MMPI, hemos encontrado un porcentaje de alteraciones psicológicas algo inferior $(54,5 \%)$ al del trabajo de Pelissier.

Del mismo modo, Subbarao (33) explora, mediante el
MMPI, una población de 45 personas afectas de una DSR y observa en un $40 \%$ de los casos un perfil psicológico alterado; siendo el componente histérico y, en menor medida, la depresión y la hipocondría las alteraciones más importantes. Para el mismo autor, la atenuación de la depresión con la mejoría del dolor confirmaría el carácter reactivo de la misma en la DSR. En el mismo sentido, Grunet (43), aplicando el MMPI en una población de 20 pacientes, encuentra tendencia a la hipocondría y a la histeria en 18 de ellos. En un trabajo anterior y también aplicando el MMPI, Wilson (44) sugiere, contrariamente a lo expuesto con anterioridad, que las alteraciones psicológicas no predisponen obligatoriamente a padecer una DSR y que cuando existe un dolor severo puede derivarse alguna alteración psicológica reactiva.

Más recientemente, Pagerols (45), aplicando el MMPI y el Inventario de Depresión de Beck, en una población de 96 pacientes diagnosticados de una DSR, encuentra que son frecuentes los antecedentes familiares de dolor crónico (46\%) e incapacidad (31\%) en la población estudiada. Entre los antecedentes personales destacan la presencia, en $1 / 3$ de los pacientes, de una síndrome depresivo en el pasado o de una infancia traumática. En el mismo estudio, la presencia de una factor estresante, relacionado temporalmente con la DSR, es el antecedente reciente más frecuentemente encontrado (72\%); siendo la pérdida afectiva el factor más significativo.

En otro sentido, Vincent y colaboradores $(34,36)$, aún admitiendo la existencia de un terreno psicológico particular en los pacientes con DSR, han puesto en entredicho la eficacia de la utilización del MMPI, alegando que este test sólo es capaz de detectar cierta tendencia de la personalidad y no la patología y el aspecto dinámico de la misma. Por otra parte, inciden en el hecho de que las alteraciones encontradas por Pelissier no son patognomónicas de la DSR, pudiéndolas encontrar en otras patologías psiquiátricas e incluso en la población normal. El autor opina que es más aconsejable el uso de una entrevista del paciente mediante psicólogo o psiquiatra, complementada con la realización del test de Rorsc harch (46).

Este cuestionario consiste en una técnica proyectiva de estilos y características de personalidad, ampliamente utilizada. Recurre a la imaginación del paciente que debe interpretar, sin límite de tiempo, imágenes informales que le son presentadas. En concreto, consta de 10 láminas con manchas de tinta simétricas. De este modo, se solicita al paciente que exprese al examinador qué le parece la mancha o bien qué le recuerda. La respuesta es registrada textualmente y puntuada mediante un sistema estandarizado.

Para cada respuesta de las láminas se analizan 4 elementos: 1) Modo de apercepción o clasificación espacial de: lámina entera $(\mathrm{G})$, detalle $(\mathrm{D})$, pequeño detalle $(\mathrm{Dd})$, detalle donde la mayoría valora una figura completa (Do), interpretación de espacios blancos entre las manchas (Dzw); 2) Determinantes o tipo de vivencia: respuesta de forma $(F)$, respuesta de color $(\mathrm{Fb})$, movimiento (B) y bien o mal vistas; 3) Contenido intrínseco: respuesta relacionada con anatomía, sexo, animales, humanos, geografía, plantas, escultura, escenas, paisajes, objetos, etc. y 4) Frecuencia estadística: respuestas originales $(\mathrm{O})$, vulgares $(\mathrm{V})$, personales, a su vez, bien o mal vistas. Se constatan también la presencia de los llamados "fenómenos especiales", a menudo típicos de determinados rasgos de personalidad, como fracasos, conciencia de interpretación, crítica del sujeto y del objeto, shock al color, shock kinestésico, 
acentuación de la asimetría, confabulaciones, perseveración, autorreferencias, etc.

En la valoración global del test se debe analizar el número de respuestas $(\mathrm{R})$, el tiempo total $(\mathrm{T})$, el tiempo de reacción, el cociente $\mathrm{R} / \mathrm{T}$, el tipo dominante de apercepción, la sucesión, el tipo de vivencia, el tipo de color, etc.

En suma, este test proporciona información muy valiosa sobre la inteligencia del sujeto, contacto con la realidad, estructura de la personalidad, mecanismos de defensa, complejos, afectividad, conflictos, etc. A través del tiempo se ha constatado la fiabilidad y validez del test pero la correcta interpretación del mismo es muy compleja y requiere notable experiencia.

En los trabajos de Vincent destaca, en la entrevista con el psicólogo, un discurso del paciente excesivamente descriptivo y la superficialidad de contacto de estos pacientes ya que difícilmente hablan de sí mismos y de su entorno. Además, se trata de individuos hiperactivos e impulsivos, con variaciones rápidas del humor y con un excesivo sentido práctico de su existencia. De la aplicación del test de Rorscharch se desprende que los pacientes con DSR evocan la psicosis y no la depresión neurótica, teniendo en cuenta que presentan destrucción de la imagen corporal, ausencia de lazos afectivos, manifestaciones emocionales inapropiadas, manifestaciones hipocondríacas y tendencia a la generalización arbritaria. Desde el punto de vista práctico, Vincent aconseja no interrogar mucho tiempo a los pacientes con DSR, no profundizar en exceso, concienciarles del aspecto benigno de su enfermedad, realizar ejercicios de relajación corporal y tratarles con algún tranquilizante y no con antidepresivos que podrían incluso aumentar su angustia. No obstante, el estudio de Vincent tiene el inconveniente de haberse realizado en una población de tan sólo 15 personas afectas de una DSR y, por tanto, sería deseable practicar este estudio con una población más amplia.

En otro trabajo, DeGood (38) realiza un estudio comparativo de los aspectos psicológicos y del comportamiento de 3 grupos de pacientes: 71 diagnosticados de una DSR, 66 de una lumbalgia crónica y 51 de una cefalea. En el estudio aplica el Symptom Checklis-90R (SCL-90R) (47).

Este test es otro ejemplo de instrumento autoinformado diseñado para proporcionar información sobre un amplio abanico de alteraciones típicas de sujetos con problemas psicológicos sintomáticos. Más breve que el MMPI, el Scl-90 R contiene sólo 90 items, puede ser administrado en 30 minutos y puntuado mediante ordenador.

Los items se combinan en 9 escalas de síntomas: 1) somatización, 2) conducta obsesiva-compulsiva, 3) sensibilidad interpersonal, 4) depresión, 5) ansiedad, 6) hostilidad, 7) ansiedad fóbica, 8) ideación paranoide y 9) psicoticismo. Se recogen, además, 3 índices globales: a) gravedad general, b) índice de síntomas positivos sobre el malestar pscológico y c) síntomas positivos totales.

La construcción de las escalas se basó en la validez de contenido y en la consistencia interna y representa un considerable esfuerzo en el desarrollo de procedimientos para la valoración psicopatológica general.

En los tres grupos estudiados por DeGood se aprecia dolor crónico y alteraciones emocionales y del comportamiento; destacando en el grupo de pacientes con DSR una mayor intensidad del dolor y de interrupciones laborables y una menor frecuencia de problemas emocionales que en los otros grupos. Al mismo tiempo, las pacientes con DSR tienen menos alteraciones de su actividad sexual y un menor uso de medicación. Por último, plantea la discusión sobre si la alteración psicológica en la DSR es causa o consecuencia.

Un enfoque relativamente nuevo para la evaluación de los trastornos de personalidad consiste en la construcción de instrumentos, ya sean autoinformes o entrevistas semiestructuradas, que valoren la presencia o ausencia de rasgos específicos de la personalidad descritos en el eje II del DSM-IV; es decir, pautas duraderas en la forma de percibir, relacionarse y pensar acerca del ambiente y sobre sí mismos que se hacen patentes en una amplia gama de contextos sociales o interpersonales. Se identifican así rasgos de personalidad que se consideran suficientemente desadaptativos e inflexibles y que causan incapacidad significativa en el funcionamiento social, laboral o personal del sujeto como para merecer la designación de "trastornos de personalidad".

Uno de los instrumentos más prometedores, en este sentido, es el Personality Diagnostic Questionnaire (PDQ) $(48,49)$. Este es un inventario autoinformado sobre los rasgos del eje-II que ofrece puntuaciones en cada una de las 13 categorías de trastornos de personalidad del DSM. Los pacientes generalmente indican un gran número de rasgos en estos instrumentos y, a menudo, reúnen los criterios para varias categorías diagnósticas; sin embargo, el PDQ puede ser útil como medida de criba.

Algunos cuestionarios evalúan la ansiedad y otros tipos de miedos relacionados con ella y resultan útiles en la clínica como medidas dimensionales de la intensidad y gravedad ansiosa o en la identificación de situaciones específicas de ansiedad. Entre ellos encontramos el State-Trait Anxiety Inventory (STAI) (50). Se trata de un cuestionario autoinformado en el que el paciente indica el grado o intensidad de su ansiedad en general (el rasgo) y en momentos puntuales (el estado); lo que posibilitará intervenciones terapeúticas específicas.

En esta línea, Geertzen (39) recurre, en otro estudio, a la aplicación conjunta del Symptom Checklist 90 R (Scl-90R), del Personality Diagnostic Questionnaire (PDQ) y del StateTrait Anxiety Inventory (STAI). En su trabajo, realizado sobre una población de 26 pacientes diagnosticados de una DSR de la mano, comparándola a un grupo control, se observa que las mujeres afectas de una DSR tienen mayor tendencia a la depresión y a la inestabilidad emocional y que el $80 \%$ de esta población ha tenido un incidente grave y reciente en su vida respecto al $20 \%$ del grupo control. El autor concluye del estudio que la depresión, la ansiedad, las alteraciones emocionales y las incidencias graves de la vida, junto al traumatismo, suponen un riesgo de aparición de la DSR.

De igual modo, Egle (31), realizando un estudio metodológico similar, hace hincapié en la tendencia a la ansiedad y a la depresión de los pacientes con DSR y encuentra que estos enfermos han tenido una agresión psíquica importante en su vida en los seis meses previos a la aparición de la DSR.

También Haddox (51) recurre a la utilización de 3 test conjuntamente: el PDQ, el STAI y el MPQ (Mc Gill Pain Questionnaire). El estudio se realiza en una población de 21 pacientes afectos de una DSR, comparándola a otra de 21 pacientes con radiculopatía; sin embargo, no encuentra diferencias psicológicas peculiares entre ambos grupos.

A modo de compendio de lo anteriormente expuesto, Lynch (15) realiza un excelente artículo de revisión sobre los aspectos psicológicos de la DSR en niños y adultos y 
repasa los diferentes trabajos publicados al respecto. De esta revisión, deduce que no hay evidencia válida para seguir manteniendo que exista una predisposición psicológica en la DSR y opina que las alteraciones psicológicas que acompañan a la DSR son probablemente consecuencia del dolor.

\section{OTROS TEST PROPUESTOS}

Teniendo en cuenta la gran controversia existente en cuanto a la aportación de los test utilizados hasta el momento en la DSR, sugerimos la utilización de otros instrumentos psicométricos de evaluación de la personalidad para la exploración de otras variables psicopatológicas y discutimos sobre sus cualidades y grado de practicidad y eficacia. Asimismo, proponemos la utilización de las modernas entrevistas clínicas estructuradas para una valoración más completa e individualizada de la situación psicosocial del paciente.

Los cuestionarios de tipo cuantitativo miden capacidades, aptitudes y rasgos de la personalidad. La selección y formulación de sus diferentes items se realiza mediante criterios de validación objetiva. Suelen ser de tipo analítico, de forma que pueden dar una idea del predominio de unas cualidades o rasgos sobre otros en la personalidad del sujeto.

Si bien uno de los más conocidos y más ampliamente aceptado a nivel internacional es el MMPI, del que ya hemos hablado, existen otros test psicométricos, también de tipo cuantitativo, validados en nuestro medio y adaptados a la población española, que tienen mayor sencillez y brevedad de formato de presentación que el MMPI y que podrían incluirse en estudios posteriores para el análisis de la personalidad en estos pacientes.

El Cuestionario Factorial de Personalidad (16 PF) (52) es considerado como uno de los mejores test factoriales de exploración de la personalidad. Se ha utilizado frecuentemente en estudios epidemiológicos y clínicos, aunque sus autores lo han orientado hacia aspectos psicológicos. Está diseñado para la validación de los constructos de la personalidad y no para la evaluación de la psicopatología.

El 16 PF explora los siguientes 16 factores que se expresan de forma polarizada:

1. Sizotimia-Ciclotimia (Reservado-Abierto).

2. Inteligencia (Pensamiento concreto-Abstracto)

3. Fuerza del yo (Emociones-Estable)

4. Sumisión-Dominancia (Conformista-Agresivo)

5. Desurgencia-Surgencia (Moderado-Impulsivo)

6. Fuerza del Superyo (Evasión de reglas-Perseverante)

7. Trectia-Parmia (Tímido-Atrevido)

8. Harria-Premsia (Duro-Sensible)

9. Alaxia-Pretensión (Confiado-Suspicaz)

10. Praxernia-Autia (Práctico-Imaginativo)

11. Sencillez-Astucia (Espontáneo-Calculador)

12. Imperturbabilidad-Culpabilidad (Tranquilo-Aprensivo)

13. Conservadurismo-Radicalismo (Tolerante-Crítico)

14. Adhesión Social-Autosuficiencia (Dependiente-Autosuficiente)

15. Poca integración-Autocontrol (Indisciplinado-Controlado)

16. Baja-Alta tensión energética (Relajado-Tenso)

Este test también permite determinar cuatro "factores de segundo orden": ansiedad, extroversión, socialización controlada e independencia. En la adaptación española se incluye, además, una escala de distorsión motivacional (DM) que expresa la tendencia del sujeto a querer ofrecer una "buena imagen personal" y otras que indican el grado de cooperación del paciente en la realización de la prueba.

El Cuestionario de Personalidad (EPQ) fue diseñado por Eysenck (53), con una versión para niños de 8 a 15 años (forma J) y otra para adultos (forma A). En España ha sido adaptado y estandarizado por Escolar (54) en 1981 y tiene amplio predicamento en nuestro medio.

El EPQ analiza cuatro dimensiones de la personalidad: neuroticismo, psicoticismo, introversión-extraversión y convencionalismo social, que han sido revalidadas en numerosos estudios y, en ocasiones, correlacionadas con datos biológicos objetivables.

La dimensión "neuroticismo" (N) define a sujetos con inestabilidad emocional, frecuentes cambios de humor, baja energía muscular y facilidad para la frustración. También se muestran frecuentemente ansiosos, preocupadizos, disfóricos y con tendencia a las quejas múltiples de tipo psicosomático.

Introversión/extraversión (I/E) define a los individuos persistentes, rígidos, irritables, subjetivos y tímidos, frente a los extravertidos que se manifiestan sociables en las relaciones interpersonales y con tendencia a la excitabilidad, vivacidad, actividad e impulsibilidad.

La dimensión psicoticismo o dureza $(\mathrm{P})$, tras el oportuno análisis factorial, resulta elevada en sujetos solitarios, problemáticos, con dificultades de empatía, poco solidarios y a veces hostiles. Con alguna frecuencia muestran inclinaciones hacia intereses raros o extravagantes, desprecian el peligro y se sienten atraídos por el riesgo.

Por último, la escala L (de "lie"-mentira) fue, inicialmente, considerada adicional y definida como índice de sinceridad para medir fiabilidad y consistencia interna del instrumento; sin embargo, los análisis posteriores definen a este factor (L) como una auténtica dimensión de la personalidad que caracteriza a sujetos normativos, inseguros, preocupados por la imagen que proyectan al exterior y excesivamente convencionales desde el punto de vista social.

La utilización de las entrevistas clínicas estructuradas o semiestructuradas, como instrumentos de evaluación psicopatológica y examen mental, representa uno de los mayores esfuerzos de la psiquiatría en las últimas décadas (55-60).

A falta de parámetros objetivos frente a los que validar nuestras impresiones clínicas, se considera por consenso internacional, que el sistema más adecuado de valoración es la entrevista amplia y el examen mental del paciente por parte de un clínico experto. La estructuración del método y la estandarización inexcusable del investigador, que se somete previamente a estudios para un mayor manejo de la entrevista y un análisis de "fiabilidad interexaminadores", favorecen la fiabilidad y validez del método.

Aunque inicialmente fueron concebidas para la investigación, estas entrevistas han demostrado gran utilidad clínica en la evaluación fiable de los criterios diagnósticos.

En este sentido, caben resaltar algunas entrevistas ya clásicas y ampliamente difundidas en nuestro medio como PSE (Wing et al, 1974), DIS (Robin et al, 1981), PAS (Dean, 1983), SICD (Spitzer \& Willians, 1985). De manera especial, destacamos CIS (Goldberg et al, 1970) (59) y EPEP (entrevista psiquiátrica estandarizada polivalente) (Lobo y cols, 1993) (60), basada en la anterior, por su utilidad clínica, aplicabili- 
dad en distintas poblaciones de enfermos y estandarización (en nuestro país por miembros de nuestro equipo de trabajoLobo y cols).

La EPEP, en especial, explora y cuantifica la presencia, frecuencia, intensidad, duración y nivel o grado de incapacidad que ocasionan numerosos síntomas y signos psicopatológicos en el paciente; tales como fatiga, trastornos del sueño, irritabilidad, depresión, ansiedad, fenómenos obsesivos, síntomas somáticos de origen psicológico presumible, fobias, despersonalización, suspicacia, histrionismo, hipocondríasis, aplanamiento afectivo, delirios, alucinaciones, deterioro intelectual, etc. En esta entrevista, se incorporan nuevas secciones como problemática social, soportes sociales y aspectos de personalidad premórbida que permiten emitir, en su caso, un diagnóstico psiquiátrico siguiendo las actuales clasificaciones internacionales: DSM-IV y CIE-10.

En suma, en la DSR interesa valorar la posible existencia de factores psicológicos que puedan influir en la aparición, desarrollo o exacerbación de la enfermedad o bien en el retraso de su recuperación. Entre estos factores pueden encontrarse trastornos mentales previos (depresión mayor, psicosis, etc); síntomas psicológicos asociados (depresión, ansiedad, etc); rasgos de personalidad o estilos de afrontamiento (negación por ejemplo) y conductas desadaptativas y perniciosas para la salud (sobreingesta, automedicación, etc).

Después de haber hecho un recorrido sobre las posibles implicaciones de las alteraciones psicológicas en la DSR, no termina de estar claro el tema en cuestión y creemos que serán necesarios nuevos trabajos para valorar, en su justa medida, si las alteraciones psicológicas asociadas a la DSR constituyen un factor predisponente o bien son reactivas a la misma. Sugerimos que estos nuevos trabajos deberían incluir: casuísticas más amplias; poblaciones más homogéneas, en cuanto a la etiología, localización y tiempo de evolución de la DSR; metodologías correctas (grupos control, estudios estadísticos) y cuestionarios más precisos y más fácilmente manejables, como hemos mencionado al final de la discusión.

No obstante, queremos recordar que en todos los casos se trata de métodos complementarios a la exploración psíquica, útiles en la investigación y que facilitan la detección ("scree ning”) y cuantificación psicopatológica, pero que deben ser siempre interpretados en el contexto global del paciente e integrarse y supeditarse a la clínica. Así, sólo una correcta anamnesis que recoja las palabras del enfermo y un detallado examen mental por parte de un clínico experto (más sencillo si se estructura el método) tiene valor diagnóstico y a veces terapeútico de primera magnitud.

En lo que respecta al reumatólogo, ante la duda de que una alteración psicológica pueda desencadenar o agravar el pronóstico de la DSR, debería remitir al especialista en psiquiatría toda persona afecta de esta patología que muestre depresión, ansiedad u otros signos de "malestar psíquico" significativo en la entrevista clínica.

\section{Bibliografía}

1. Herisson C, Claustre J, Simon L. Les algodystrophies sympathiques réflexes. Prat Med 1985; 38: 7-29.

2. Bruscas C. Aportación de las exploraciones vasculares, isotópicas y de la absorciometría bifotónica al conocimiento de la algodistrofia (Tesis). Facultad Zaragoza 199; 567p

3. Bruscas C, Barjon MC, Simon L. Aportación de las exploraciones isotópicas al conocimiento de la algodistrofia. Rev Esp Reumatol 1992; 19 : 138-141.

4. Doury P, Dirheimer Y, Pattin S. Algodystrophy: diagnosis and therapy of a frequent disease of the locomotor apparatus. Part 5, Springler-Verlag, Berlin, 1981; 102-103.

5. Kozin F, Ryan LM, Carrera GF, Soin JS. The reflex sympahetic dystrophy syndrome. Am J Med 1981; 70: 23-30.

6. Bruscas C, Medrano M, Simon L. El diagnóstico de la distrofia simpático refleja. An Med Interna (Madrid) 1999; 16: 308-314

7. Leriche R. Oèdeme dur post-traumatique de la main avec impotence fonctionnelle complète. Transformation soudain 5 heures après sympathectomie humérale. Lyon Chir 1923; 20: 814-818.

8. Dotson RM. Causalgia-reflex sympathetic dystrophy-sympathectically maintained pain: myth and reality. Muscle Nerve 1993; 16: 1049-1055.

9. Scott GD. An unsympathetic view of pain. Lancet 1995; 345: 634-636.

10. Stanton-Hicks M, Jänig W, Hassenbusch S, Haddox JD, Boas R, Wilson P. Reflex sympathetic dystrophy: changing concepts and taxonomy. Pain 1995; 52: 127-133

11. Berthelot JM, Glemarec J, Guillot P, Mangars Y, Prost A. Algodystrophies-causalgies: nouveaux concepts nosologiques, physiopathologiques et pathogéniques. Rev Rhum Mal Osteoartic 1997; 64: 562-573.

12. Acquaviva P, Schiano A, Harnden P, Cros D, Serratrice G. Les algodystrophies, terrain et facteurs pathogéniques. Résultats d' une enquête multicentrique portant sur 765 observations. Rev Rhum Mal Osteoartic 1982; 49: 761-766.

13. Bruscas C, Simon L. Algodistrofia: terreno favorecedor y factores

desencadenantes. An Med Intern (Madrid) 1992 : 9: 401-405.

14. Bruscas Izu C, Jiménez Aznárez A, Sancho Serrano MA, Simon L. Algodistrofia de cadera y embarazo. An Med Intern (Madrid) 1992; 9: 134-136.

15. Lynch ME. Psychological aspects of reflex sympathetic dystrophy: a review of the adult and paediatric literature. Pain 1992; 49: 337-347.

16. Rodríguez de la Serna A. Características psicosomáticas más relevantes de los pacientes reumáticos. En: Angera de Sojo I. Medicina Psicosomática. Ed Doyma. Barcelona, 1988; 132-139.

17. Matles AC. Reflex sympathetic dystrophy in a child: a case report. Bull Hosp Joint Dis Orthop Inst 1971; 32: 193-197.

18. Fermaglich DR. Reflex sympathetic dystrophy in children. Pediatrics 1977; 60: 881-883

19. Bernstein BH, Singsen BH, Kent JT et al. Reflex neurovascular dystrophy in childhood. Pediatrics 1978; 93: 211-215.

20. Ruggeri SB, Athreya BH; Doughty R, Gregg JR, Das MM. Reflex sympathetic dystrophy in children. Clin Orthop 1982; 163: 225-230.

21. Forster RS, Fu FH. Reflex sympathetic dystrophy in children. Orthopedics 1985 ; 8: 475-477.

22. Sherry DD, Weisman R. Psychologic aspects of childhood reflex neurovascular dystrophy. Pediatrics 1988; 81: 572-578.

23. Doury P, Pattin S, Gaillard F, Eulry F, Pattin C. L'algodystrophie de l'enfant. Ann Pediatr 1988; 35: 469-475.

24. Bruscas Izu C, Polo Ostáriz MA, Simon L. Algodistrofia del niño y del adolescente: aspectos comparativos con la del adulto. An Med Intern (Madrid) 1995; 12: 197-200.

25. Lidz T, Payne RL. Causalgia report of recovery following relief of emotional stress. Arch Neuropsych 1945; 1: 222-225.

26. Adler E, Weiss AA, Zonari D. A psychosomatic approach to sympathetic reflex dystrohy. Psychiatr Neurol 1959; 138: 256-271.

27. Zachariae L. Incidence and course of post-traumatic dystrophy following operation for Dupuytren's contracture. Acta Chir Scand 1964; 336 (Suppl): 6-51. 
28. Pak TJ, Martin GM, Magness JL, Kavanaugh GJ. Reflex sympathetic dystrophy: review of 140 cases. Minn Med 1970: 53: 507-512.

29. Pollack HJ, Newmann R, Pollack EM. Südeck and psyche. Bietr Orthoped Traumatol 1980; 27: 463-468.

30. Van Houdenhove B. Neuro-algodystrophy: a psychiatrist' view. Clin Rheumatol 1986; 5: 399-406.

31. Egle UT, Hoffman SO. Psychosomatische zysammenhänge bei sympathischer reflexdystrophie (morbus Südeck). Literaturubersicht und erste klinische ergebnisse. Psychother Psychosom Med Psychol 1990; 40: 123-135.

32. Pelissier J, Touchon J, Besset A et al. La personnalité du sujet atteint d'algodystrophie sympathique réflexe. Etude psychométrique par le test MMPI. Rhumatologie 1981; 23: 351-354.

33. Subbarao J, Stillwell GK. Reflex sympathetic dystrophy syndrome of the upper extremity: analysis of total outcome of management of 125 cases. Arch Phys Med Rehabil 1981; 62: 549-554.

34. Vincent G, Ernst J, Henniaux M, Beaubigny M. Essai d'approche psychologique dans les algoneurodystrophies. Rev Rhum Mal Osteoartic 1982; 49: 767-769.

35. Pelissier J, Touchon J, Chartier J, Besset J, Simon L. La personnalité du sujet atteint d'algodystrophie. Evaluation psychométrique par le MMPI. En: Simon L, Herisson C, eds. Acquisitions rhumatologiques: les algodystrophies sympathiques réflexes. Paris Masson, 1987; 113-118.

36. Vincent G, Ernst J, Heniaux M, Beaubigny M. Les malades atteints d'algoneurodystrophie ont-ils un profil psychologique particulier? En: Simon L, Herisson C, eds. Acquisitions rhumatologiques: les algoneurodystrophies sympathiques réflexes. Paris Masson; 1987; 119-123.

37. Zucchini M, Alberti G, Moretti MP. Algodystrophy and related psychological features. Funct Neurol 1989; 4: 153-156.

38. DeGood DE, Cundiff GW, Adams LE, Shutty MS Jr. A psychosocial and behavioral comparison of reflex sympathetic dystrophy, low back pain, and headache patients. Pain 1993; 54: 317-322.

39. Geertzen JHB, De Bruijn H, De Bruijn-Kofman AT, Arendzen JH Reflex sympathetic dystrophy: early treatment and psychological aspects. Arch Phys Med Rehabil 1994; 75: 442-446.

40. Hathaway SR, Mc Kinley JC. Minnesota Multiphasic Personality Inventory Manual (MMPI). Minneapolis. University of Minnesota Press, 1943. Revised Edition. New York, Psychological Corporation, 1967

41. Pheasant HC, Gilbert D, Gold-Farb J, Herron L. The MMPI as a predictor of outcome in low-back pain. Spine 1979; 4: 78-84.

42. Rook JC, Pesch RN, Keeler EC. Chronic pain and the questionable use of the Minnesota Multiphasic Personality Inventory. Arch Phys Med Rehabil 1981; 62: 373-376.

43. Grunert BK, Devine CA, Sanger JR, Matloub HS, Green A. Thermal self-regulation for pain control in reflex sympathetic dystrophy syndro- me. J Hand Surg 1990; 15: 615-618.

44. Wilson RL. Management of pain following peripheral nerve injuries. Orthop Clin North Am 1981; 12: 343-359.

45. Pagerols Bonilla M. Síndrome de dolor regional complejo tipo I: aspectos psicosociales. Rev Esp Reumatol 1998; 25: 265-269.

46. Rorscharch H. Psychodiagnostics. New York, Grune and Stratton, 1949

47. Derogatis LR, Clearly PA. Confirmation of the dimensional structure of the Scl-90: A study in construct validation. J Clin Psychol, 1977; 33: 981-989.

48. Hurt SW, Hyler SE, Frances A et al. Assessing borderline personality disorder with self-report, clinical interview, or semiestructured interview. Am J Psychiatry 1984; 141: 1228-1231.

49. Hyler SE, Rieder R, Spitzer RL et al. Personality diagnostic questionnaire (PDQ). New York. New York State Psychiatric Institute. Biometrics Research Division, 1978.

50. Spielberg CD, Gorsuch RL, Luchene RE. Manual for the State-Trait Anxiety Inventory. Palo Alto, CA. Consulting Psychologists Press, 1976.

51. Haddox JD, Abram SE. Hopwood MH. Comparison of psychometric data in RSD and radiculopathy. Reg Anesth 1988; 13: 27.

52. Cattell RB, Eber HW, Tatsuoka MM. Handbook for the Sixteen Personality Factor Inventory. Champaign, IL. Institute for personality and ability testing, 1970 .

53. Eysenck HJ, Eysenck SB. The structure and measurement of personality. RR Knapp, San Diego CA, 1969.

54. Escolar V. Aportaciones del uso del cuestionario de Eysenck (EPQ) para adultos en la población española. Com. Psiquiátrica VIII, 267-331, 1981.

55. Wing J, Cooper J, Sartorius N. The description and classification of psychiatric symtoms: An instruction manual for the PSE and CATEGO system. London University Press, 1974

56. Dean C, Surtees PG, Sashidharan SP. Comparison of research diagnostic systems in a Edimburgh community sample. Br J Psychiatry 1983; 142: 247-256.

57. Spitzer R, Willians JB. Structured clinical interview for DSM-III R New York. New York Psychiatric Institute, 1985

58. Robins LN, Helzer JE, Croughan J, Ratcliff KS. National institute of mental health diagnostic interview schedule: it's history, characteristics and validity. Arch Gen Psych 1991; 38: 381-389.

59. Goldberg P, Cooper B, Eastwood MR, Kedward HB, Sheperd M. A standarized psychiatric interview for use in community survery. $\mathrm{Br} \mathrm{J}$ Prev Soc Med 1970; 24: 18-23.

60. Lobo A, Campos R, Pérez-Echeverría MJ, Izuzquiza J, García-Mayayo JJ. A new interview for the multiaxial assesment of psychiatric morbidity in medical settings. Psychological Med 1993; 23: 505-510. 